\title{
Magnetocaloric Effect in Geometrically Frustrated Magnetic Compound $\mathrm{HoB}_{12}$
}

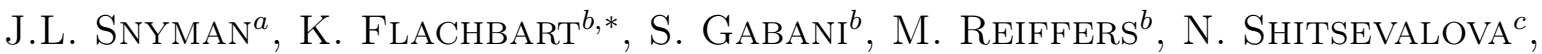 \\ K. SiemensmeyeR ${ }^{d}$ AND A.M. StRYdom ${ }^{a}$ \\ ${ }^{a}$ Physics Department, University of Johannesburg, P.O. Box 524, Auckland Park, 2006, South Africa
}

${ }^{b}$ Institute of Experimental Physics, Slovak Academy of Sciences, Watsonova 47, SK-04001 Kosice, Slovak Republic ${ }^{c}$ I. Frantsevich Inst. for Problems of Materials Science of NASU, 3 Krzhyzhanovsky Str., UA-03680 Kiev, Ukraine

${ }^{d}$ Helmholtz Zentrum Berlin, Glienicker Str. 100, D-14109 Berlin, Germany

\begin{abstract}
The magnetocaloric effect of frustrated antiferromagnetic $\mathrm{HoB}_{12}$ is calculated. The isothermal entropy change $\Delta S$ characterising the magnetocaloric effect shows a small positive change upon magnetisation below the transition temperature $T_{\mathrm{N}}$, indicating a small inverse magnetocaloric effect. At $T_{\mathrm{N}}, \Delta S$ shows clear scaling behaviour with the applied magnetic field. The adiabatic temperature change $\Delta T$ shows paramagnetic behaviour above $T_{\mathrm{N}}$, despite strong magnetic correlations that persist in this temperature region. The adiabatic temperature change calculated for $\mathrm{HoB}_{12}$ is appreciable.
\end{abstract}

PACS numbers: 75.30.Sg, 75.40.Cx

\section{Introduction}

The magnetocaloric effect (MCE) in materials represents a magneto-thermodynamic phenomenon in which a change of temperature or entropy is achieved by an adiabatic or isothermal change of magnetic field [1]. As magnetic correlations limit the lowest temperature which may be achieved through magnetic cooling, paramagnetic salts have historically been used as magnetic refrigerants.

In this work we present first magnetocaloric studies of holmium dodecaboride $\mathrm{HoB}_{12}$, which is a frustrated incommensurate amplitude-modulated antiferromagnet (AFM) with weak crystal field anisotropy emerging in high magnetic fields [2-4]. The paramagnetic Weiss temperature of $\mathrm{HoB}_{12}$ is $\Theta_{\mathrm{W}}=-24.3 \mathrm{~K}$ and its Néel temperature $T_{\mathrm{N}}=7.4 \mathrm{~K}$. Neutron scattering patterns observed above $T_{\mathrm{N}}$ indicate that below $\left|\Theta_{\mathrm{W}}\right|$, pronounced short range magnetic correlations appear between neighbouring Ho-ions, similar to low-dimensional magnets in a cooperative paramagnetic state $[5,6]$. The magnetic entropy locked up in the spin sublattice over such a broad temperature interval is conceivably of importance to systems of this materials class and thus $\mathrm{HoB}_{12}$ appears to be a very interesting and suitable candidate for magnetocaloric studies in geometrically frustrated magnetic systems.

\section{Results and discussion}

\subsection{Isothermal entropy change}

The synthesis and characterization of $\mathrm{HoB}_{12}$ samples, as well as details of the measurements of which the results

\footnotetext{
* corresponding author; e-mail: flachb@saske.sk
}

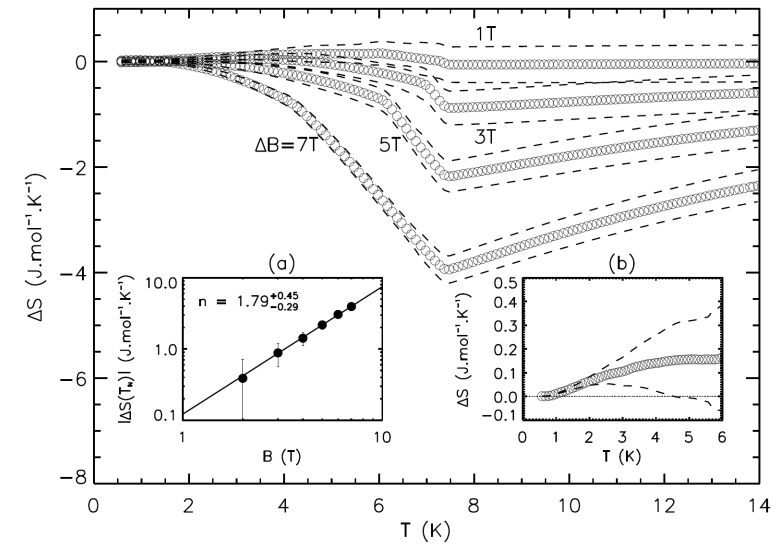

Fig. 1. The isothermal entropy change $\Delta S$ upon magnetisation calculated for $\mathrm{HoB}_{12}$. Dashed lines indicate margins of error. Inset (a): $\left|\Delta S\left(T_{\mathrm{N}}\right)\right|$ for various applied fields on a double-log plot to emphasize the observed power-law behaviour. Inset (b): enlargement of $\Delta S$ for $\Delta B=1 \mathrm{~T}$ showing the inverse MCE below $T=T_{\mathrm{N}}$.

are analyzed in this work have been discussed elsewhere [2-4]. Shown in Fig. 1 is the isothermal entropy change upon magnetisation calculated from previously reported heat capacity data $[3,7]$. Values are obtained utilising the indirect method discussed in [1]. The numerical error inherent to this method could be constrained by noting that the zero field magnetic entropy thus calculated saturates within $3 \%$ of the $R \ln 3$ value expected for $\mathrm{HoB}_{12}$. The initial applied field is taken to be zero throughout, so that $\Delta B=B_{\mathrm{f}}\left(B_{\mathrm{f}}\right.$ is the applied field which is taken as $B=\mu_{0} H$ for simplicity).

Below $T_{\mathrm{N}}$ a positive value for $\Delta S$ is found for applied fields up to $5 \mathrm{~T}$, which implies an inverse MCE in this 
field range, consistent with other AFM compounds [8]. The scaling behaviour of $\Delta S$ at a magnetic phase transition was recently investigated by Franco et al. [9]. In the critical region it is expected that

$$
|\Delta S| \sim B^{n} \text { where } n=1+\frac{1}{\delta}\left(1-\frac{1}{\beta}\right) .
$$

Here $\delta$ and $\beta$ are the critical exponents describing the dependences of the order parameter on the applied field and reduced temperature, respectively. The inset of Fig. 1 shows the absolute value of the $\Delta S$ at $T_{\mathrm{N}}$ for different applied fields. The values of $\left|\Delta S\left(T_{\mathrm{N}}\right)\right|$ clearly show the expected scaling behaviour (Eq. (2.1)) with $n=1.79_{-0.29}^{+0.45}$. Standard models of magnetism predict values of $n<1[10]$. For $\mathrm{HoB}_{12} n>1$ implies that $\Delta S / \Delta B$ increases with increasing $\Delta B$ up to at least $7 \mathrm{~T}$.

\subsection{Adiabatic temperature change}

The calculated adiabatic temperature change $\Delta T$ upon demagnetisation is shown in Fig. 2. $T_{\mathrm{i}}$ corresponds to the temperature at which the sample is adiabatically isolated in a nonzero applied field $B_{\mathrm{i}}$ before letting $B \rightarrow 0$. Calculations presented in this section neglect the lattice contribution to the heat capacity.

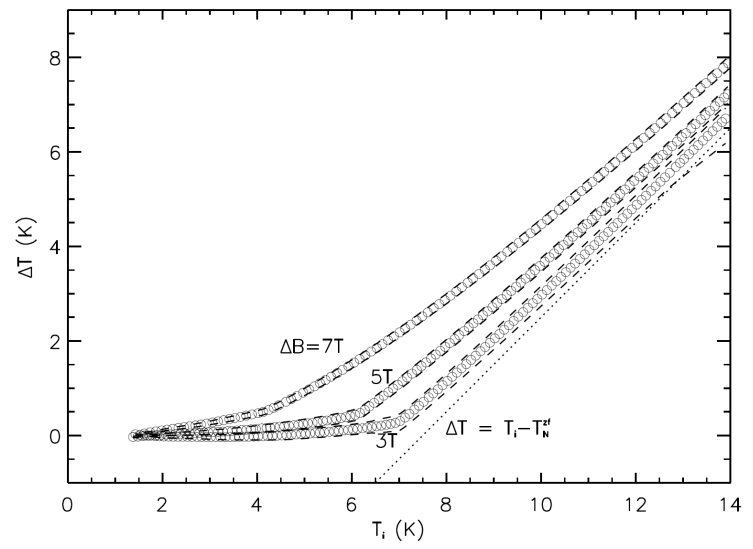

Fig. 2. The adiabatic temperature change $\Delta T$ upon demagnetisation. Dashed lines indicate margins of error. The dotted line indicates the MCE expected above a second order phase transition for $\Delta B \rightarrow 0$. $T_{\mathrm{N}}^{\mathrm{zf}}$ is the Néel temperature in zero applied magnetic field.

Immediately above $T_{\mathrm{N}}, \Delta T$ becomes linear in $T_{\mathrm{i}}$. Despite the strong magnetic correlations that persist in this region $\Delta T$ shows paramagnetic behaviour. Below $T_{\mathrm{N}}$ a small inverse magnetocaloric effect is observed, consistent with the results for $\Delta S$ reported above. Including the entropy contribution from the lattice does not produce an MCE that differs significantly from the results shown in Fig. 2. The lattice contribution to the heat capacity is very small at $T=14 \mathrm{~K}\left(\approx 0.6 \mathrm{~J} \mathrm{~mol}^{-1} \mathrm{~K}^{-1}\right.$. See [7]). Although not shown here, the lattice contribution does not alter the paramagnetic behaviour seen above $T_{\mathrm{N}}$. Generally, $\Delta T$ is significantly reduced due to the lattice contribution in the region where $S\left(B_{\mathrm{i}}, T_{\mathrm{i}}\right)>R \ln (2 J+1)$. This condition is not satisfied for $B_{\mathrm{i}} \geq 3 \mathrm{~T}$ and $T_{\mathrm{i}}$ up to $14 \mathrm{~K}$ in $\mathrm{HoB}_{12}$. The result is a large, almost paramagnetic $\mathrm{MCE}$ in the region above $7.4 \mathrm{~K}$.

\section{Conclusion}

The magnetocaloric effect in $\mathrm{HoB}_{12}$ is calculated from heat capacity data. In the ordered region a small inverse magnetocaloric effect is observed. At $T_{\mathrm{N}}$ the isothermal entropy change shows clear scaling behaviour with applied field. The value of the exponent that describes this behaviour differs from values predicted by standard models of magnetic systems. Despite short range magnetic correlations, the behaviour of the adiabatic temperature change $\Delta T$ above $T_{\mathrm{N}}$ is asymptotically close to the behaviour expected for an ideal paramagnet, and yields appreciable changes in temperature upon adiabatic demagnetisation.

\section{Acknowledgments}

J.L.S. thanks the University of Johannesburg as well as the SA-NRF for funding under the New Generation Scholarship Program and the DoL Scarce Skills bursary program. K.F. and M.R. acknowledge the support of the Slovak Research and Development Agency under contract APVV - SK-ZA-0009-07. A.M.S. thanks the SA-NRF for funding under a SA-Slovakia research grant (67873).

\section{References}

[1] V.K. Pecharsky, K.A. Gschneidner, Jr., J. Appl. Phys. 86, 565 (1999).

[2] S. Gabani, I. Batko, K. Flachbart, T. Herrmannsdoerfer, R. Koenig, Y. Paderno, N. Shitsevalova, J. Magn. Magn. Mater. 207, 131 (1999).

[3] A. Kohout, I. Batko, A. Czopnik, K. Flachbart, S. Matas, M. Meissner, Y. Paderno, N. Shitsevalova, K. Siemensmeyer, Phys. Rev. B 70, 224416 (2004).

[4] K. Siemensmeyer, K. Habicht, Th. Lonkai, S. Mat'as, S. Gabani, N. Shitsevalova, E. Wulf, K. Flachbart, J. Low Temp. Phys. 146, 581 (2007).

[5] J. Villian, Z. Phys. B, Condens. Matter 33, 31 (1979).

[6] J.S. Gardner, S.R. Dunsiger, B.D. Gaulin, M.J.P. Gingras, J.E. Greedan, R.F. Kiefl, M.D. Lumsden, W.A. MacFarlane, N.P. Raju, I. Swainson, J.E. Sonier, Z. Tun, Phys. Rev. Lett. 82, 1012 (1999).

[7] A. Czopnik, N. Shitsevalova, A. Krivchikov, V. Pluzhnikov, Y. Paderno, Y. Onuki, J. Solid State Chem. 177, 507 (2004).

[8] J.H. Schelleng, S.A. Friedberg, Phys. Rev. 185, 728 (1969).

[9] V. Franco, J.S. Blazquez, A. Conde, Appl. Phys. Lett. 89, 222512 (2006).

[10] L.P. Kadanof, W. Gotze, D. Hamblen, R. Hecht, E.A.S. Lewis, V.V. Palciauskas, M. Rayl, J. Swift, Rev. Mod. Phys. 39, 395 (1967). 